Supporting information

\title{
Total Synthesis of Marine Sponge Bis(indole) Alkaloids of Topsentin Class
}

\author{
Xavier Guinchard, Yannick Vallée and Jean-Noël Denis*
}

\section{Table of contents}

General methods

Experimental procedure and characterisation data for 11a

Experimental procedure and characterisation data for $\mathbf{4 a}$

Experimental procedure and characterisation data for $\mathbf{1 1 b}$

Experimental procedure and characterisation data for $\mathbf{4 b}$

Experimental procedure and characterisation data for 11c

Experimental procedure and characterisation data for $\mathbf{4 c}$

Experimental procedure and characterisation data for $\mathbf{2} \mathbf{b}$

Experimental procedure and characterisation data for $\mathbf{2 c}$

Experimental procedure and characterisation data for 1d

Experimental procedure and characterisation data for $\mathbf{1 e}$

${ }^{1} \mathrm{H}$ and ${ }^{13} \mathrm{C}$ NMR spectra for $\mathbf{4 a}$

${ }^{1} \mathrm{H}$ and DEPT ${ }^{13} \mathrm{C}$ NMR spectra for $\mathbf{4 b}$

${ }^{1} \mathrm{H}$ and DEPT ${ }^{13} \mathrm{C}$ NMR spectra for $\mathbf{4 c}$

${ }^{1} \mathrm{H}$ and ${ }^{13} \mathrm{C}$ NMR spectra for $\mathbf{8 a}$

${ }^{1} \mathrm{H}$ and ${ }^{13} \mathrm{C}$ NMR spectra for $\mathbf{2 a}$

${ }^{1} \mathrm{H}$ NMR spectrum for $\mathbf{2 b}$

${ }^{1} \mathrm{H}$ and ${ }^{13} \mathrm{C}$ NMR spectra for $\mathbf{2 c}$

${ }^{1} \mathrm{H}$ and DEPT ${ }^{13} \mathrm{C}$ NMR spectra for $\mathbf{1 a}$

${ }^{1} \mathrm{H}$ and DEPT ${ }^{13} \mathrm{C}$ NMR spectra for $\mathbf{1 d}$

${ }^{1} \mathrm{H}$ and DEPT ${ }^{13} \mathrm{C}$ NMR spectra for $1 \mathrm{e}$ 


\section{General methods}

${ }^{1} \mathbf{H}$ NMR, ${ }^{13} \mathbf{C}$ and DEPT ${ }^{13} \mathbf{C}$ NMR spectra were recorded at 300 and $75.5 \mathrm{MHz}$ respectively. Chemical shifts are given in $\mathrm{ppm}(\delta)$ and were referenced to the internal solvent signal or to TMS used as an internal standard. Multiplicities are declared as follow: $s$ (singlet), br $s$ (broad singlet), $d$ (doublet), $t$ (triplet), $q$ (quadruplet), quint (quintuplet), sept (septet), $d d$ (doublet of doublet), $d d d$ (doublet of doublet of doublet), $d t$ (doublet of triplet), $m$ (multiplet). Coupling constants $J$ are given in Hz.

Infrared spectra (IR) were obtained either as neat films on sodium chloride discs or in potassium bromide plates. Data are reported in reciprocal centimetres $\left(\mathrm{cm}^{-1}\right)$.

Melting points were uncorrected.

LRMS mass spectra were recorded using DCI $\left(\mathrm{NH}_{3}+\right.$ isobutane) or ESI+ (ion polarity: positive) as ion sources. HRMS mass spectra were recorded with ESI+ as ion source.

Reactions were performed using oven dried glasswares under an atmosphere of dry argon. They were monitored by thin layer chromatography (TLC) using commercial aluminiumbacked silica gel plates. TLC spots were viewed under ultraviolet light and by heating the plate after treatment with an appropriate revelatory (ninhydrin, $\mathrm{KMnO}_{4}, \mathrm{TTC}$ ).

Solvents: Tetrahydrofuran and diethyl ether were refluxed on sodium-benzophenone and then distilled. Dichloromethane and methanol were dried by refluxing on $\mathrm{CaH}_{2}$ and then distilled. Unless otherwise noted, all reagent-grade chemicals and solvents were obtained from commercial suppliers and were used as received. 
1-(indol-3'-yl)-2-(piperidin-1-yl)-2-thioxoethanone (11a). To a solution of $2 \mathrm{~g}$ (17.1 mmol) of indole $3 \mathbf{a}$ in anhydrous diethyl ether $(35 \mathrm{~mL})$ at $0{ }^{\circ} \mathrm{C}$, oxalyl chloride $(1.72 \mathrm{~mL}$, $19.8 \mathrm{mmol}$ ) was added dropwise over $30 \mathrm{~min}$. The reaction mixture was stirred at $0{ }^{\circ} \mathrm{C}$ for $3 \mathrm{~h}$ and then allowed to warm to rt during an additional hour. The resulting yellow crystals were collected by filtration, washed with cold anhydrous diethyl ether and dried under vacuum. The yellow solid 10a has been used without further purification (1.63 g, $15.7 \mathrm{mmol}, 92 \%)$. 10a (3.07 g, $14.8 \mathrm{mmol})$ was dissolved into $15 \mathrm{~mL}$ of EtOAc at $0{ }^{\circ} \mathrm{C}$. A solution of tributyltin hydride $(3.98 \mathrm{~mL}, 14.82 \mathrm{mmol})$ in $20 \mathrm{~mL}$ of EtOAc was then added. The reaction mixture was stirred at $0{ }^{\circ} \mathrm{C}$ for $30 \mathrm{~min}$, warmed to rt and then stirred for an additional night. Hexane was added and the resulting yellow powder was collected by filtration. Washing with a large amount of hexane and drying under vacuum gave the corresponding ketoaldehyde $(2.038 \mathrm{~g}$, $11.78 \mathrm{mmol}, 79 \%)$ which was used without further purification. The ketoaldehyde (600 mg, $3.49 \mathrm{mmol}$ ) was dissolved into $3.5 \mathrm{~mL}$ of pyridine. Sulfur was then added (139 mg, 4.36 mmol) followed by piperidine (445 $\mathrm{mg}, 5.24 \mathrm{mmol}$ ). The resulting mixture was warmed to 80 ${ }^{\circ} \mathrm{C}$ during $5 \mathrm{~h}$. It was then poured in water. Aqueous layer was extracted three times by EtOAc. The combined organic layers were washed with brine, dried over anhydrous $\mathrm{MgSO}_{4}$, filtered and evaporated under vacuum. Pyridine excess was azeotropically evaporated with toluene. The obtained solid was purified by column chromatography on silica gel (Eluent: EtOAc/Pentane, 1:1). The resulting thioamide 11a was collected as brown solid and recristallised in methanol to give brown crystals (365 mg, $1.34 \mathrm{mmol}, 38 \%$; $30 \%$ from 10a). Mp: $158-159{ }^{\circ} \mathrm{C} . \mathrm{IR}$ (neat): $3269,2942,2856,1601,1520,1507,1438,1246,1176,1131 \mathrm{~cm}^{-}$ 1. ${ }^{1} \mathrm{H} \mathrm{NMR}\left(\mathrm{CDCl}_{3}, 300 \mathrm{MHz}\right): \delta=1.35-1.85(\mathrm{~m}, 6 \mathrm{H}), 3.53(\mathrm{t}, J=5.1$ and $5.6 \mathrm{~Hz}, 2 \mathrm{H}), 4.19(\mathrm{t}$, $J=4.7$ and $5.6 \mathrm{~Hz}, 2 \mathrm{H}), 7.1-7.3(\mathrm{~m}, 2 \mathrm{H}), 7.39(\mathrm{dd}, J=2.2$ and $6.9 \mathrm{~Hz}, 1 \mathrm{H}), 7.85(\mathrm{~s}, 1 \mathrm{H}), 8.22$ $(\mathrm{d}, J=6.7 \mathrm{~Hz}, 1 \mathrm{H}), 10.16(\mathrm{~s}, 1 \mathrm{H}) .{ }^{13} \mathrm{C} \mathrm{NMR}\left(\mathrm{CDCl}_{3}, 75.5 \mathrm{MHz}\right): \delta=23.8,25.3,26.4,48.3$, $52.9,112.4,113.2,121.5,122.9,123.9,125.2,135.5,136.7,185.6,195.0$. LRMS (DCI, 
$\mathrm{NH}_{3}+$ isobutane): $\mathrm{m} / \mathrm{z}=273\left[(\mathrm{M}+\mathrm{H})^{+}\right]$. HRMS (ESI) calcd for $\mathrm{C}_{15} \mathrm{H}_{17} \mathrm{~N}_{2} \mathrm{OS}: 273.1062$. Found: 273.1071 $\left[(\mathrm{M}+\mathrm{H})^{+}\right]$. Anal. calcd for $\mathrm{C}_{15} \mathrm{H}_{16} \mathrm{~N}_{2} \mathrm{OS}$ : C, 66.15; H, 5.92, N, 10.28. Found: $\mathrm{C}$, 66.40, H, 5.95, N, 10.30.

\section{1-[2-(indol-3'-yl)-1-methylsulfanyl-2-oxo-ethylidene]-piperidinium iodide (4a).}

Thioamide 11a (200 mg, $0.735 \mathrm{mmol})$ was dissolved into $0.95 \mathrm{~mL}$ of methyl iodide (20 equiv). The mixture was refluxed during $5 \mathrm{~h}$, cooled at $\mathrm{rt}$ and excess of methyl iodide was evaporated under vacuum. The thioimidate salt $\mathbf{4 a}$ was obtained as a white unstable solid (295 mg, $0.72 \mathrm{mmol}$ ), which was immediately used without further purification. Yield: $98 \%$. ${ }^{1} \mathrm{H}$ NMR $\left(\mathrm{CD}_{3} \mathrm{OD}, 300 \mathrm{MHz}\right): \delta=1.70-2.10(\mathrm{~m}, 5 \mathrm{H}), 2.10-2.20(\mathrm{~m}, 1 \mathrm{H}), 2.65(\mathrm{~s}, 3 \mathrm{H}), 3.85-$ $4.00(\mathrm{~m}, 1 \mathrm{H}), 4.00-4.10(\mathrm{~m}, 1 \mathrm{H}), 4.15-4.30(\mathrm{~m}, 2 \mathrm{H}), 7.30-7.45(\mathrm{~m}, 2 \mathrm{H}), 7.55-7.65(\mathrm{~m}, 1 \mathrm{H})$, 8.20-8.38 (m, 1H), $8.63(\mathrm{~s}, 1 \mathrm{H}) .{ }^{13} \mathrm{C}$ NMR $\left(\mathrm{CD}_{3} \mathrm{OD}, 75.5 \mathrm{MHz}\right): \delta=16.8,23.4,26.8,28.1$, $55.9,58.7,114.1,114.3,122.6,125.3,125.8,126.5,139.2,140.3,176.8,186.0$.

1-(6'-bromoindol-3'-yl)-2-(piperidin-1-yl)-2-thioxoethanone (11b). To a solution of 500 $\mathrm{mg}(2.55 \mathrm{mmol})$ of 6-bromoindole in anhydrous diethyl ether $(5 \mathrm{~mL})$ at $0{ }^{\circ} \mathrm{C}$ was added dropwise oxalyl chloride $(0.25 \mathrm{~mL}, 2.93 \mathrm{mmol})$ over $30 \mathrm{~min}$. The reaction mixture was stirred at $0{ }^{\circ} \mathrm{C}$ for $3 \mathrm{~h}$, and then allowed to warm to $23{ }^{\circ} \mathrm{C}$ during an additional hour. The resulting yellow crystals were collected by filtration, washed with cold anhydrous diethyl ether and dried under vacuum. The yellow solid 10b $(600 \mathrm{mg}, 2.09 \mathrm{mmol}, 82 \%)$ has been used without further purification. 10b $(740 \mathrm{mg}, 2.58 \mathrm{mmol})$ was dissolved into $2.6 \mathrm{~mL}$ of EtOAc at $0{ }^{\circ} \mathrm{C}$. A solution of tributyltin hydride $(0.69 \mathrm{~mL}, 2.58 \mathrm{mmol})$ in $3.8 \mathrm{~mL}$ of EtOAc was then added. The reaction mixture was stirred at $0{ }^{\circ} \mathrm{C}$ for $30 \mathrm{~min}$, warmed to $\mathrm{rt}$ and then stirred for an additional night. Hexane was added and the resulting yellow powder was collected by filtration. Washing with a large amount of hexane and drying under vacuum gave the ketoaldehyde (453 mg, $1.79 \mathrm{mmol}, 69 \%$ ) which was used without further purification. The ketoaldehyde (453 mg, $1.79 \mathrm{mmol}$ ) was dissolved into $2 \mathrm{~mL}$ of pyridine. Sulfur was then 
added $(58 \mathrm{mg}, 1.79 \mathrm{mmol})$ followed by piperidine $(0.27 \mathrm{~mL}, 229 \mathrm{mg}, 2.69 \mathrm{mmol})$. The resulting mixture was warmed to $80{ }^{\circ} \mathrm{C}$ during $5 \mathrm{~h}$. It was then poured into water. Aqueous layer was extracted three times by EtOAc. Organic layers were washed with brine, dried over anhydrous $\mathrm{MgSO}_{4}$ and evaporated under vacuum. Excess pyridine was azeotropically evaporated with toluene. The obtained solid was purified by column chromatography on silica gel (Eluent: $\mathrm{Et}_{2} \mathrm{O}$ ). The product 11b was then obtained as a yellow solid (280 $\mathrm{mg}, 0.798$ mmol, $45 \%$; $30 \%$ from 10b) and recrystallized in methanol. Mp: $242-243{ }^{\circ} \mathrm{C}$. IR (neat): 3160, 2940, 2914, 2856, 1712, 1622, 1512, 1421, 1247, 1176, $1143 \mathrm{~cm}^{-1} .{ }^{1} \mathrm{H}$ NMR (DMSO, $300 \mathrm{MHz}): \delta=1.4-1.6(\mathrm{~m}, 2 \mathrm{H}), 1.6-1.8(\mathrm{~m}, 4 \mathrm{H}), 3.55(\mathrm{t}, J=5.1$ et $5.6 \mathrm{~Hz}, 2 \mathrm{H}), 4.18(\mathrm{br} \mathrm{s}, 2 \mathrm{H})$, $7.38(\mathrm{dd}, J=1.5$ et $8.5 \mathrm{~Hz}, 1 \mathrm{H}), 7.72(\mathrm{~d}, J=1.8 \mathrm{~Hz}, 1 \mathrm{H}), 8.02(\mathrm{~d}, J=8.5 \mathrm{~Hz}, 1 \mathrm{H}), 8.12(\mathrm{~s}, 1 \mathrm{H})$.

${ }^{13} \mathrm{C}$ NMR (DMSO, $\left.75.5 \mathrm{MHz}\right): \delta=23.3,24.9,26.2,47.5,52.3,112.4,115.3,115.8,122.5$, 124.2, 125.2, 136.8, 137.7, 184.2, 193.8. LRMS (DCI, $\mathrm{NH}_{3}+$ isobutane): $\mathrm{m} / \mathrm{z}=351$ and 353 $\left[(\mathrm{M}+\mathrm{H})^{+}\right]$. HRMS (ESI) Calcd for $\mathrm{C}_{15} \mathrm{H}_{15}{ }^{79} \mathrm{BrN}_{2} \mathrm{NaOS}: 372.9986$. Found: 372.9988 $\left[(\mathrm{M}+\mathrm{Na})^{+}\right]$. Anal. calcd for $\mathrm{C}_{15} \mathrm{H}_{15} \mathrm{BrN}_{2} \mathrm{OS}: \mathrm{C}, 51.29 ; \mathrm{H}, 4.30 ; \mathrm{Br}, 22.75 ; \mathrm{N}, 7.97$. Found: $\mathrm{C}$, 51.26; H, 4.37; Br, 22.80; N, 7.74.

\section{1-[2-(6'-bromoindol-3'-yl)-1-methylsulfanyl-2-oxo-ethylidene]-piperidinium}

iodide

(4b). The thioamide $11 \mathbf{b}(213 \mathrm{mg}, 0.605 \mathrm{mmol})$ was dissolved into $0.77 \mathrm{~mL}$ of methyl iodide (20 eq). The mixture was refluxed during $5 \mathrm{~h}$, cooled at $\mathrm{rt}$ and methyl iodide was removed under vacuum. The product $\mathbf{4 b}$ was collected as a yellow unstable solid (296 $\mathrm{mg}, 0.60 \mathrm{mmol}$ ) which was immediately used without further purification. Yield: $100 \%$. ${ }^{1} \mathrm{H}$ NMR $\left(\mathrm{CD}_{3} \mathrm{OD}\right.$, $300 \mathrm{MHz}): \delta=1.65-2.05(\mathrm{~m}, 5 \mathrm{H}), 2.05-2.25(\mathrm{~m}, 1 \mathrm{H}), 2.65(\mathrm{~s}, 3 \mathrm{H}), 3.85-4.00(\mathrm{~m}, 1 \mathrm{H}), 4.0-4.1$ (m, 1H), 4.1-4.3 (m, 2H), $7.49(\mathrm{dd}, J=1.7$ and $8.5 \mathrm{~Hz}, 1 \mathrm{H}), 7.78(\mathrm{~d}, J=1.4 \mathrm{~Hz}, 1 \mathrm{H}), 8.14(\mathrm{~d}$, $J=8.5 \mathrm{~Hz}, 1 \mathrm{H}), 8.68(\mathrm{~s}, 1 \mathrm{H}) . \mathrm{DEPT}{ }^{13} \mathrm{C} \mathrm{NMR}\left(\mathrm{CD}_{3} \mathrm{OD}, 75.5 \mathrm{MHz}\right): \delta=16.9,23.4,26.8,28.1$, $56.0,58.8,114.2,117.1,119.6,124.0,124.7,128.4,140.0,141.0,177.0,185.7$. 
1-(6'-methoxyindol-3'-yl)-2-piperidin-1-yl-2-thioxoethanone (11c). To a solution of 250 $\mathrm{mg}(1.7 \mathrm{mmol})$ of 6-methoxyindole in anhydrous diethyl ether $(2.5 \mathrm{~mL})$ at $0{ }^{\circ} \mathrm{C}$ was added dropwise oxalyl chloride $(0.17 \mathrm{~mL}, 2.6 \mathrm{mmol})$ over $30 \mathrm{~min}$. The reaction mixture was stirred at $0{ }^{\circ} \mathrm{C}$ for $3 \mathrm{~h}$, and then allowed to warm to $23{ }^{\circ} \mathrm{C}$ during an additional hour. The obtained brown solid 10c (331 mg, $1.2 \mathrm{mmol}, 71 \%$ ) has been used without further purification. 10c (900 mg, $3.79 \mathrm{mmol}$ ) was dissolved into $3.8 \mathrm{~mL}$ of EtOAc at $0{ }^{\circ} \mathrm{C}$. A solution of tributyltin hydride $(10.1 \mathrm{~mL}, 3.79 \mathrm{mmol})$ in $5.6 \mathrm{~mL}$ of EtOAc was then added. The reaction mixture was stirred at $0{ }^{\circ} \mathrm{C}$ for $30 \mathrm{~min}$, warmed to $\mathrm{rt}$ and then stirred for an additional night. Hexane was added and the resulting brown powder was collected by filtration. Washing with a large amount of hexane and drying under vacuum gave the ketoaldehyde as a brown solid (780 mg, $3.84 \mathrm{mmol}$, quant.) which was used without further purification. The ketoaldehyde (780 mg, $3.84 \mathrm{mmol}$ ) was dissolved into $4 \mathrm{~mL}$ of pyridine. Sulfur was then added (122 $\mathrm{mg}, 3.84 \mathrm{mmol}$ ) followed by piperidine $(0.57 \mathrm{~mL}, 490 \mathrm{mg}, 5.76 \mathrm{mmol})$. The resulting mixture was warmed to $80^{\circ} \mathrm{C}$ during $5 \mathrm{~h}$. It was then poured into water. Aqueous layer was extracted three times by EtOAc. Organic layers were washed with brine, dried over anhydrous $\mathrm{MgSO}_{4}$ and evaporated under vacuum. Excess pyridine was azeotropically evaporated with toluene. The obtained oil was purified by column chromatography on silica gel (Eluent: $\mathrm{Et}_{2} \mathrm{O}$ ). The product 11c was then obtained as a brown solid (384 mg, $1.27 \mathrm{mmol}, 33 \%$; 33\% from 10c). Mp: $124-126{ }^{\circ} \mathrm{C}$. IR (KBr): 3268, 2957, 2932, 2854, 1604, 1579, 1517, 1501, 1443, 1248, 1157, $1021 \mathrm{~cm}^{-1} .{ }^{1} \mathrm{H}$ NMR $\left(\mathrm{CD}_{3} \mathrm{OD}, 300 \mathrm{MHz}\right): \delta=1.50-1.62(\mathrm{~m}, 2 \mathrm{H}), 1.68-1.85(\mathrm{~m}, 4 \mathrm{H}), 3.58(\mathrm{t}, J=4.6$ and 6.0 $\mathrm{Hz}, 2 \mathrm{H}), 3.82(\mathrm{~s}, 3 \mathrm{H}), 4.23(\mathrm{t}, J=4.6$ and $6.0 \mathrm{~Hz}, 2 \mathrm{H}), 6.89(\mathrm{dd}, J=2.3$ and $8.7 \mathrm{~Hz}, 1 \mathrm{H}), 6.99$ $(\mathrm{d}, J=2.3 \mathrm{~Hz}, 1 \mathrm{H}), 7.89(\mathrm{~s}, 1 \mathrm{H}), 8.01(\mathrm{dd}, J=0.4$ and $8.7 \mathrm{~Hz}, 1 \mathrm{H}) .{ }^{13} \mathrm{C} \mathrm{NMR}\left(\mathrm{CD}_{3} \mathrm{OD}, 75.5\right.$ MHz): $\delta=25.0,26.4,27.6,49.22,54.0,56.0,96.5,113.5,114.4,120.7,123.0,136.6,139.5$, 159.0, 187.6, 196.5. LRMS (ESI): $\mathrm{m} / \mathrm{z}=303\left[(\mathrm{M}+\mathrm{H})^{+}\right]$. HRMS (ESI) Calcd for $\mathrm{C}_{16} \mathrm{H}_{19} \mathrm{~N}_{2} \mathrm{O}_{2} \mathrm{~S}$ : 
303.1167. Found: $303.1168\left[(\mathrm{M}+\mathrm{H})^{+}\right]$. Anal. calcd for $\mathrm{C}_{16} \mathrm{H}_{18} \mathrm{~N}_{2} \mathrm{O}_{2} \mathrm{~S}: \mathrm{C}, 63.55 ; \mathrm{H}, 6.00 ; \mathrm{N}$, 9.26. Found: C, 63.61; H, 6.14; N, 9.11.

\section{1-[2-(6'-methoxyindol-3'-yl)-1-methylsulfanyl-2-oxo-ethylidene]-piperidinium iodide}

(4c). The thioamide $11 \mathrm{c}(178 \mathrm{mg}, 0.59 \mathrm{mmol})$ was dissolved into $0.75 \mathrm{~mL}$ of methyl iodide (20 eq). The mixture was refluxed during $5 \mathrm{~h}$. The crude mixture was then cooled at $\mathrm{rt}$ and methyl iodide was removed under vacuum. The product $\mathbf{4 c}$ was obtained as a brown unstable solid (260 mg, $0.59 \mathrm{mmol}$ ) which was immediately used without further purification. Yield: $100 \% .{ }^{1} \mathrm{H}$ NMR $\left(\mathrm{CD}_{3} \mathrm{OD}, 300 \mathrm{MHz}\right): \delta=1.7-2.0(\mathrm{~m}, 5 \mathrm{H}), 2.05-2.20(\mathrm{~m}, 1 \mathrm{H}), 2.63(\mathrm{~s}, 3 \mathrm{H})$, $3.84(\mathrm{~s}, 3 \mathrm{H}), 3.90-4.10(\mathrm{~m}, 2 \mathrm{H}), 4.20(\mathrm{t}, J=5.7$ and $5.5 \mathrm{~Hz}, 2 \mathrm{H}), 6.96(\mathrm{dd}, J=2.3$ and $8.7 \mathrm{~Hz}$, 1H), $7.13(\mathrm{~d}, J=2.3 \mathrm{~Hz}, 1 \mathrm{H}), 8.04(\mathrm{~d}, J=8.7 \mathrm{~Hz}, 1 \mathrm{H}), 8.57$ (s, $1 \mathrm{H}) . \mathrm{DEPT}^{13} \mathrm{C}$ NMR $\left(\mathrm{CD}_{3} \mathrm{OD}\right.$, 75.5 MHz): $\delta=17.1,23.3,26.7,28.0,55.8,56.3,58.6,97.2,114.2,114.9,119.2,123.1$, $139.6,140.1,159.8,176.4,185.8$

Spongotine B (2b). To a stirred solution of indolic diamine dihydrochloride 8a $(50 \mathrm{mg}$, $0.20 \mathrm{mmol})$ and thioimidate iodide $4 \mathbf{b}(100 \mathrm{mg}, 0.20 \mathrm{mmol})$ in distilled methanol $(1 \mathrm{~mL})$ was added Amberlyst A21 (200 mg). The resulting mixture was stirred for $60 \mathrm{~h}$ at rt. Methanol was then removed under vacuum. To the residue was added water and ethyl acetate. The aqueous layer was extracted three times with EtOAc. The combined organic layers were then washed with brine and dried over anhydrous $\mathrm{MgSO}_{4}$. After the removal of the solvent, the residue was purified by column chromatography on silica gel (Eluent: EtOAc/pentane, 9:1). Spongotine B 2b was obtained as a brown solid (38 mg, $0.09 \mathrm{mmol})$. Yield: 46 \%. Mp: 250 ${ }^{\circ} \mathrm{C}$. IR (KBr): $2850,1615,1570,1518,1454,1369,1253,1169,827 \mathrm{~cm}^{-1} .{ }^{1} \mathrm{H}$ NMR $\left(\mathrm{CD}_{3} \mathrm{OD}\right.$, $300 \mathrm{MHz}): 3.90(\mathrm{dd}, J=8.6$ and $12.2 \mathrm{~Hz}, 1 \mathrm{H}), 4.23(\mathrm{t}, J=11,9 \mathrm{~Hz}, 1 \mathrm{H}), 5.45(\mathrm{dd}, J=8.6$ and $11.3 \mathrm{~Hz}, 1 \mathrm{H}), 7.00(\mathrm{dt}, J=1.0$ and $7.2 \mathrm{~Hz}, 1 \mathrm{H}), 7.11(\mathrm{dt}, J=1.0$ and $7.0 \mathrm{~Hz}, 1 \mathrm{H}), 7.25(\mathrm{~s}, 1 \mathrm{H})$, 7.32-7.43 (m, 1H), $7.63(\mathrm{~d}, J=1,7 \mathrm{~Hz}, 1 \mathrm{H}), 7.67(\mathrm{~d}, J=1.7 \mathrm{~Hz}, 1 \mathrm{H}), 8.00(\mathrm{~s}, 1 \mathrm{H}), 8.09$ (d, $J=$ $8.6 \mathrm{~Hz}, 1 \mathrm{H}), 8.20(\mathrm{~d}, J=8.6 \mathrm{~Hz}, 1 \mathrm{H}), 8.52(\mathrm{~s}, 1 \mathrm{H}) .{ }^{13} \mathrm{C} \mathrm{NMR}\left(\mathrm{CD}_{3} \mathrm{OD}, 75.5 \mathrm{MHz}\right): \delta=57.5$, 
$59.5,112.7,115.7,116.2,118.0,118.2,119.9,120.0,122.7,123.5,124.3,126.3,126.8,126.9$, 138.8, 139.2, 139.8, 164.3, 182.1. LRMS (DCI, $\mathrm{NH}_{3}+$ isobutane): $\mathrm{m} / \mathrm{z}=407$ and 409 $\left[(\mathrm{M}+\mathrm{H})^{+}\right]$. HRMS (ESI) calcd for $\mathrm{C}_{20} \mathrm{H}_{16}{ }^{79} \mathrm{BrN}_{4} \mathrm{O}: 407.0507$. Found: $407.0525\left[(\mathrm{M}+\mathrm{H})^{+}\right]$.

\section{6'-methoxyindol-3'-yl-[5-(indol-3''-yl)-4,5-dihydroimidazol-2-yl] ketone (2c). To a} stirred solution of indolic diamine dihydrochloride $8 \mathbf{3 a}(280 \mathrm{mg}, 1.14 \mathrm{mmol})$ and thioimidate iodide $4 \mathbf{c}(504 \mathrm{mg}, 1.14 \mathrm{mmol})$ in distilled methanol $(4 \mathrm{~mL})$ was added amberlyst A21 $(1.1 \mathrm{~g})$. The resulting mixture was stirred for $60 \mathrm{~h}$ at $\mathrm{rt}$. Methanol was then removed under vacuum. The resulting residue was purified by column chromatography on silica gel (eluant: EtOAc). The $\alpha$-ketoimidazoline $2 \mathrm{c}$ was obtained as a yellow solid $(256 \mathrm{mg}, 0.72 \mathrm{mmol}$ ). Yield: $63 \%$. Mp: $230{ }^{\circ} \mathrm{C}$ (dec). IR (KBr): 3399, 3166, 2927, 1634, 1589, 1576, 1525, 1447, 1421, 1201, 1163, $1092 \mathrm{~cm}^{-1} .{ }^{1} \mathrm{H}$ NMR $\left(\mathrm{CD}_{3} \mathrm{COCD}_{3}, 300 \mathrm{MHz}\right): 3.81$ (s, 3H,), 3.99 (dd, $J=8.8$ and 12.1 $\mathrm{Hz}, 1 \mathrm{H}), 4.39(\mathrm{t}, J=12.1 \mathrm{~Hz}, 1 \mathrm{H}), 5.58(\mathrm{dd}, J=8.8$ and $11.0 \mathrm{~Hz}, 1 \mathrm{H}), 6.90(\mathrm{dd}, J=2.3$ and 8.7 $\mathrm{Hz}, 1 \mathrm{H}), 7.01(\mathrm{t}, J=7.1$ and $7.9 \mathrm{~Hz}, 1 \mathrm{H}), 7.07-7.17(\mathrm{~m}, 2 \mathrm{H}), 7.39(\mathrm{~d}, J=2.1 \mathrm{~Hz}, 1 \mathrm{H}), 7.45$ (d, $J=8.1 \mathrm{~Hz}, 1 \mathrm{H}), 7.65(\mathrm{~d}, J=7.8 \mathrm{~Hz}, 1 \mathrm{H}), 8.19(\mathrm{~d}, J=8.7 \mathrm{~Hz}, 1 \mathrm{H}), 8.87$ (s, 1H), $10.27(\mathrm{~s}, 1 \mathrm{H}$, $\mathrm{NH}$ indol.), 11.29 (s, $1 \mathrm{H}, \mathrm{NH}$ indol.). ${ }^{13} \mathrm{C} \mathrm{NMR}\left(\mathrm{CD}_{3} \mathrm{COCD}_{3}, 75.5 \mathrm{MHz}\right): \delta=55.8,57.0$, $58.5,96.4,112.6,113.1,115.2,117.3,119.8,119.9,121.1,122.5,123.4,123.6,126.6,138.2$ 138.4, 138.6, 158.4, 163.2, 178.5. LRMS (ESI): $\mathrm{m} / \mathrm{z}=359\left[(\mathrm{M}+\mathrm{H})^{+}\right]$. HRMS (ESI) calcd for $\mathrm{C}_{21} \mathrm{H}_{19} \mathrm{~N}_{4} \mathrm{O}_{2}: 359.1508$. Found: $359.1504\left[(\mathrm{M}+\mathrm{H})^{+}\right]$.

Isobromodeoxytopsentin (1d). IBX $(0.05 \mathrm{mmol}, 14 \mathrm{mg})$ was added to a solution of spongotine B $\mathbf{2 b}(18 \mathrm{mg}, 0.044 \mathrm{mmol})$ in $0.2 \mathrm{~mL}$ of DMSO. The resulting mixture was stirred at $\mathrm{rt}$ during $15 \mathrm{~h}$ to completion. It was then quenched by addition of a saturated aqueous solution of $\mathrm{Na}_{2} \mathrm{~S}_{2} \mathrm{O}_{3}(0.11 \mathrm{~mL})$ and an equal volume of EtOAc. The mixture was treated with a saturated aqueous solution of $\mathrm{NaHCO}_{3}$. Aqueous layer was then extracted two times by EtOAc. Combined organic layers were washed with an aqueous saturated solution of $\mathrm{NaHCO}_{3}$, with brine and then dried over anhydrous $\mathrm{MgSO}_{4}$. After the removal of the solvent, 
the residue was purified by column chromatography on silica gel (Eluent: EtOAc/pentane, 1:1). The isobromodeoxytopsentine $\mathbf{1 d}$ was obtained as a yellow solid (6 $\mathrm{mg}, 0.015 \mathrm{mmol})$. Yield: 34 \%. Mp: $246{ }^{\circ} \mathrm{C}$ (dec). IR (neat): 3431, 3283, 1699, 1596, 1518, 1454, 1240, $859 \mathrm{~cm}^{-}$ 1. ${ }^{1} \mathrm{H}$ NMR $\left(\mathrm{CD}_{3} \mathrm{COCD}_{3}, 500 \mathrm{MHz}\right)$ : isomer $a: \delta=7.10-7.26(\mathrm{~m}, 2 \mathrm{H}), 7.40(\mathrm{dd}, J=1.0$ and $8.0 \mathrm{~Hz}, 1 \mathrm{H}), 7.47(\mathrm{~d}, J=8.0 \mathrm{~Hz}, 1 \mathrm{H}), 7.74(\mathrm{~s}, 1 \mathrm{H}), 7.79$ (d, $J=1.0 \mathrm{~Hz}, 1 \mathrm{H}), 7.85(\mathrm{~d}, J=2.0$ Hz, 1H), 8.22 (d, J=7.5 Hz, 1H), 8.45 (d, J=7.5 Hz, 1H), 9.62 (s, 1H), 10.38 (s, 1H), 11.24 (s, 1H), $12.10(\mathrm{~s}, 1 \mathrm{H}) . \underline{\text { isomer } b}: \delta=7.10-7.26(\mathrm{~m}, 2 \mathrm{H}), 7.38(\mathrm{dd}, J=1.5$ and $8.0 \mathrm{~Hz}, 1 \mathrm{H}), 7.52$ $(\mathrm{d}, J=8.0 \mathrm{~Hz}, 1 \mathrm{H}), 7.63(\mathrm{~s}, 1 \mathrm{H}), 7.77(\mathrm{~d}, J=1.5 \mathrm{~Hz}, 1 \mathrm{H}), 7.96(\mathrm{~d}, J=7.0 \mathrm{~Hz}, 1 \mathrm{H}), 8.08$ (d, $J=$ $2.0 \mathrm{~Hz}, 1 \mathrm{H}), 8.42$ (d, J= 7.5 Hz, 1H), 9.40 (s, 1H), 10.64 (s, 1H), 11.20 (s, 1H), 12.18 (s, 1H). ${ }^{1} \mathrm{H}$ NMR $\left(\mathrm{CD}_{3} \mathrm{COCD}_{3}+\mathrm{TFA}-d+\mathrm{D}_{2} \mathrm{O}, 500 \mathrm{MHz}\right): \delta=7.21(\mathrm{t}, J=7.0$ and $8.0 \mathrm{~Hz}, 1 \mathrm{H}), 7.26$ (t, $J=7.0$ and $8.0 \mathrm{~Hz}, 1 \mathrm{H}), 7.45(\mathrm{dd}, J=1.5$ and $8.0 \mathrm{~Hz}, 1 \mathrm{H}), 7.56(\mathrm{~d}, J=8.0 \mathrm{~Hz}, 1 \mathrm{H}), 7.82(\mathrm{~d}$, $J=2.0 \mathrm{~Hz}, 1 \mathrm{H}), 7.96(\mathrm{~d}, J=7.5 \mathrm{~Hz}, 1 \mathrm{H}), 8.07(\mathrm{~s}, 1 \mathrm{H}), 8.12(\mathrm{~s}, 1 \mathrm{H}), 8.26(\mathrm{~d}, J=8.0 \mathrm{~Hz}, 1 \mathrm{H})$, $8.87(\mathrm{~s}, 1 \mathrm{H}) . \mathrm{DEPT}{ }^{13} \mathrm{C} \mathrm{NMR}\left(\mathrm{CD}_{3} \mathrm{COCD}_{3}, 75.5 \mathrm{MHz}\right)$ : Isomers $a$ and $b: \delta=111.9,112.4$, $112.8,115.3,115.7,115.8,116.7,116.8,120.3,120.4,121.1,121.2,122.4,123.1,123.3$, $124.4,124.4,124.5,125.7,125.8,126.0,126.4,126.5,127.1,127.3,131.3,132.6,137.8$, $137.58,137.9,137.95, \quad 138.2,138.35,138.3,138.7,140.4,176.9$. DEPT ${ }^{13} \mathrm{C}$ NMR $\left(\mathrm{CD}_{3} \mathrm{COCD}_{3}\right.$, TFA- $\left.d, \mathrm{D}_{2} \mathrm{O}, 75.5 \mathrm{MHz}\right): \delta=102.9,113.2,114.9,116.5,118.2,119.7,121.8$ $123.7,124.0,125.4,125.9,126.5,127.1,132.4,137.7,138.7,138.9,141.3,171.7$. LRMS (ESI): $\mathrm{m} / \mathrm{z}=405$ and $407\left[(\mathrm{M}+\mathrm{H})^{+}\right]$. HRMS (ESI) calcd for $\mathrm{C}_{20} \mathrm{H}_{14}{ }^{79} \mathrm{BrN}_{4} \mathrm{O}: 405.0351$. Found: $405.0345\left[(\mathrm{M}+\mathrm{H})^{+}\right]$.

$\boldsymbol{O}$-methyltopsentin (1e). IBX (0.04 mmol, $12 \mathrm{mg})$ was added to a solution of 4,5-dihydro-O-methyltopsentin $2 \mathrm{c}(13 \mathrm{mg}, 0.036 \mathrm{mmol})$ in $0.2 \mathrm{~mL}$ of DMSO. The resulting mixture was stirred at $\mathrm{rt}$ during $15 \mathrm{~h}$ to completion. It was then quenched by addition of a saturated aqueous solution of $\mathrm{Na}_{2} \mathrm{~S}_{2} \mathrm{O}_{3}(0.1 \mathrm{~mL})$ and an equal volume of EtOAc. The mixture was treated with a saturated aqueous solution of $\mathrm{NaHCO}_{3}$. Aqueous layer was then extracted 
two times by EtOAc. Combined organic layers were washed with an aqueous saturated solution of $\mathrm{NaHCO}_{3}$, with brine and then dried over anhydrous $\mathrm{MgSO}_{4}$. After the removal of the solvent, the residue was purified by column chromatography on silica gel (Eluent: EtOAc). The $O$-methyltopsentin 1e was obtained as a yellow solid (13 mg, $0.036 \mathrm{mmol})$. Yield: 100 \%. IR (KBr): 3394, 2956, 2918, 2848, 1733, 1716, 1519, 1456, 1254, $1158 \mathrm{~cm}^{-1}$. ${ }^{1} \mathrm{H}$ NMR $\left(\mathrm{CD}_{3} \mathrm{COCD}_{3}, 300 \mathrm{MHz}\right)$ : isomer $a: \delta=3.85(\mathrm{~s}, 3 \mathrm{H}), 6.87-6.95(\mathrm{~m}, 1 \mathrm{H}), 7.07-7.13$ (m, 1H), 7.13-7.30 (m, 2H), 7.45-7.50 (m, 1H), $7.70(\mathrm{~d}, J=2.1 \mathrm{~Hz}, 1 \mathrm{H}), 7.83(\mathrm{~d}, J=2.4 \mathrm{~Hz}$, 1H), 8.20-8.26 (m, 1H), 8.37 (d, $J=8.7 \mathrm{~Hz}, 1 \mathrm{H}), 9.49$ (d, $J=3.0 \mathrm{~Hz}, 1 \mathrm{H}), 10.35(\mathrm{~s}, 1 \mathrm{H}), 10.90$ $(\mathrm{s}, 1 \mathrm{H}), 12.05(\mathrm{~s}, 1 \mathrm{H}) . \underline{\text { isomer } b}: \delta=3.84(\mathrm{~s}, 3 \mathrm{H}), 6.87-6.95(\mathrm{~m}, 1 \mathrm{H}), 7.07-7.13(\mathrm{~m}, 1 \mathrm{H}), 7.13-$ $7.30(\mathrm{~m}, 2 \mathrm{H}), 7.50-7.55(\mathrm{~m}, 1 \mathrm{H}), 7.60(\mathrm{~s}, 1 \mathrm{H}), 7.93-7.99(\mathrm{~m}, 1 \mathrm{H}), 8.07(\mathrm{~d}, J=2.5 \mathrm{~Hz}, 1 \mathrm{H})$, $8.36(\mathrm{~d}, J=8.7 \mathrm{~Hz}, 1 \mathrm{H}), 9.27$ (d, $J=2.8 \mathrm{~Hz}, 1 \mathrm{H}), 10.60(\mathrm{~s}, 1 \mathrm{H}), 10.88(\mathrm{~s}, 1 \mathrm{H}), 11.98(\mathrm{~s}, 1 \mathrm{H})$. ${ }^{1} \mathrm{H}$ NMR $\left(\mathrm{CD}_{3} \mathrm{COCD}_{3}+\mathrm{TFA}-d+\mathrm{D}_{2} \mathrm{O}, 300 \mathrm{MHz}\right): \delta=3.84(\mathrm{~s}, 3 \mathrm{H}), 6.96(\mathrm{dd}, J=2.3$ and 8.7 $\mathrm{Hz}, 1 \mathrm{H}), 7.16(\mathrm{~d}, J=2.1,1 \mathrm{H}), 7.20(\mathrm{dt}, J=1.5$ and $7.0 \mathrm{~Hz}, 1 \mathrm{H}), 7.25(\mathrm{dt}, J=1.7$ and $7.0 \mathrm{~Hz}$, 1H), 7.54-7.59 (m, 1H), 7.90-7.96 (m, 1H), $8.05(\mathrm{~s}, 1 \mathrm{H}), 8.11(\mathrm{~s}, 1 \mathrm{H}), 8.17(\mathrm{dd}, J=0.5$ and 8.7 $\mathrm{Hz}, 1 \mathrm{H}), 8.60(\mathrm{~s}, 1 \mathrm{H}) . \mathrm{DEPT}{ }^{13} \mathrm{C} \mathrm{NMR}\left(\mathrm{CD}_{3} \mathrm{COCD}_{3}\right.$, TFA- $\left.d, \mathrm{D}_{2} \mathrm{O}, 75.5 \mathrm{MHz}\right): \delta=55.7,96.6$, $103.5,113.0,113.5,114.9,116.6,119.7,121.5,121.8,123.1,123.4,125.4,126.1,132.5$, 137.6, 140.0, 141.9, 158.7, 172.1. LRMS (ESI): $\mathrm{m} / \mathrm{z}=357\left[(\mathrm{M}+\mathrm{H})^{+}\right]$. HRMS (ESI) calcd for $\mathrm{C}_{21} \mathrm{H}_{16} \mathrm{~N}_{4} \mathrm{O}_{2}: 367.1352$. Found: $367.1365\left[(\mathrm{M}+\mathrm{H})^{+}\right]$. 


$$
t^{n}=1
$$



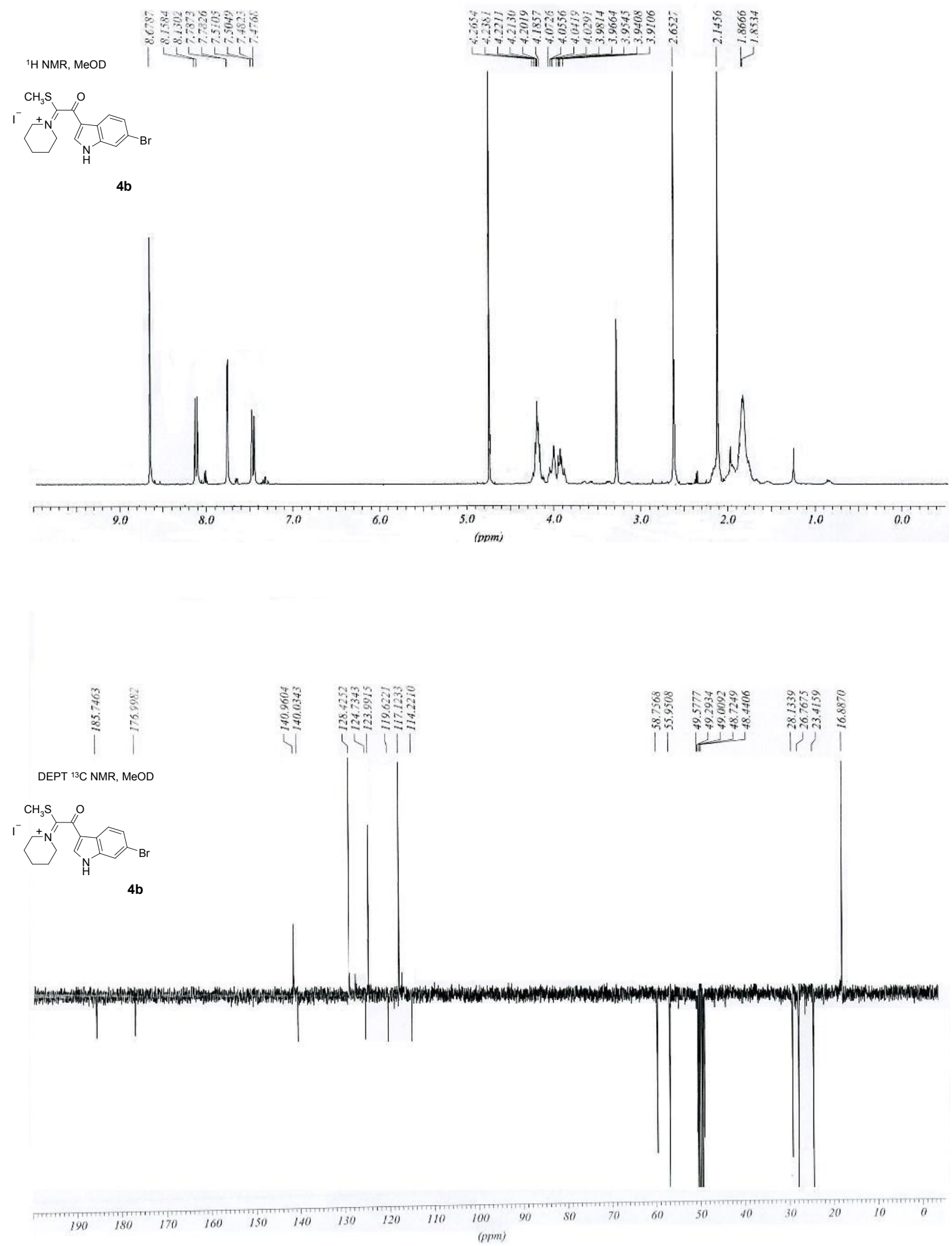

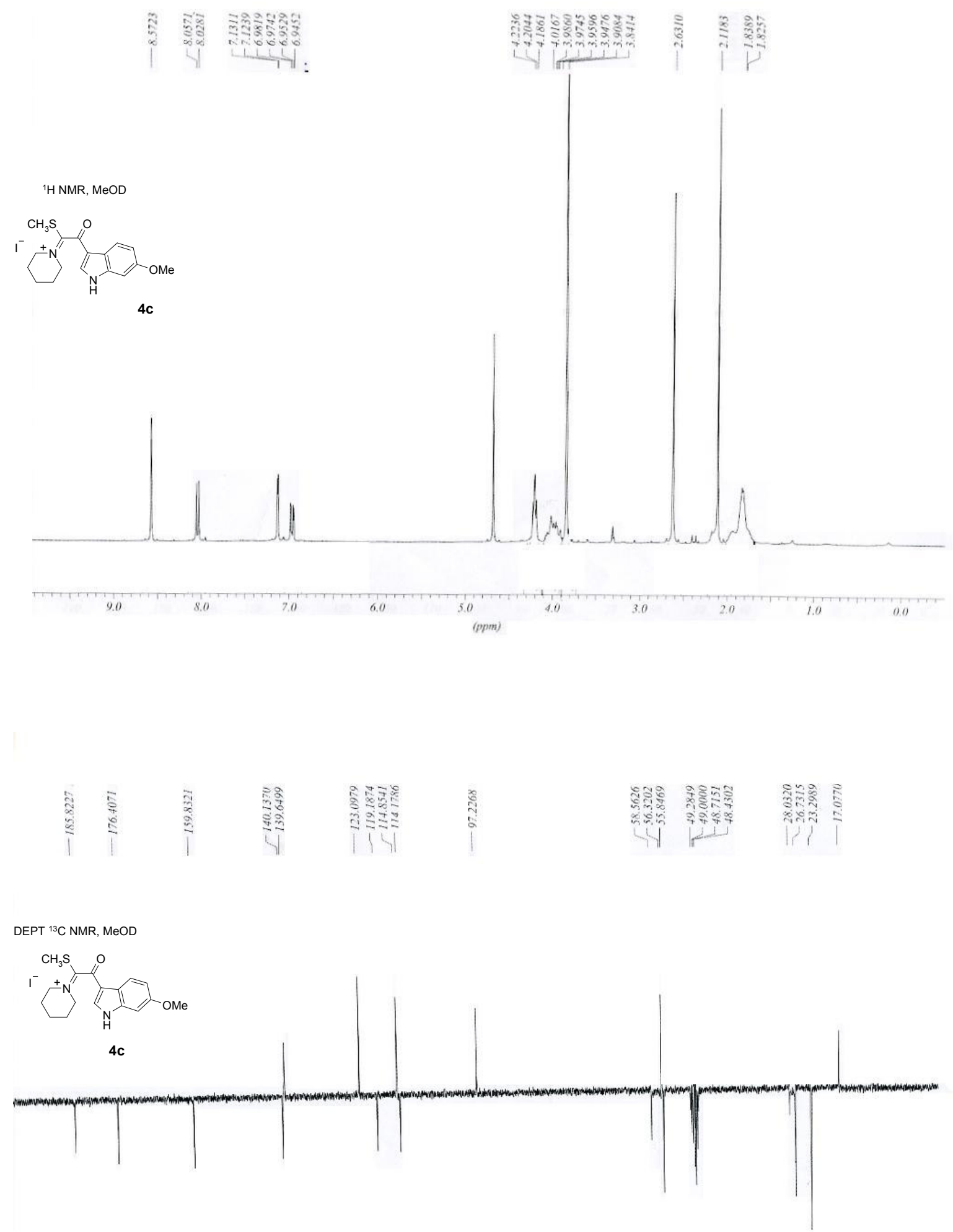

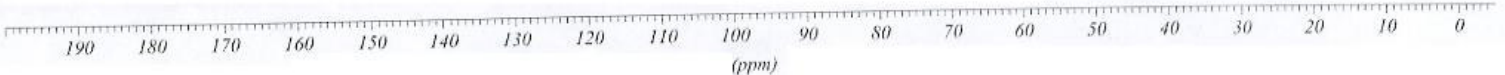




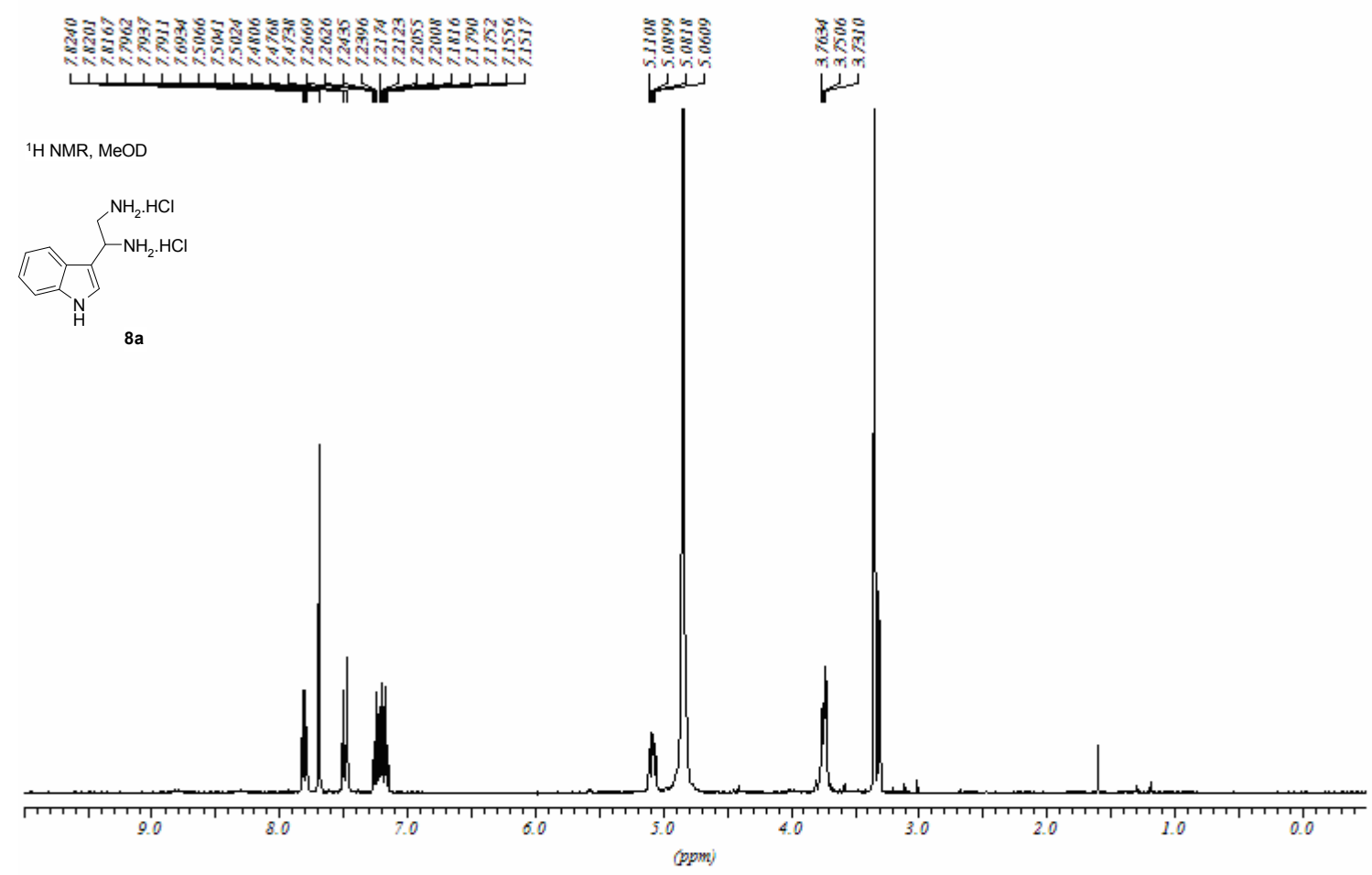

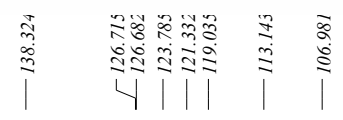

${ }^{13} \mathrm{C} \mathrm{NMR}, \mathrm{MeOD}$

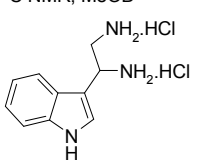

$8 a$

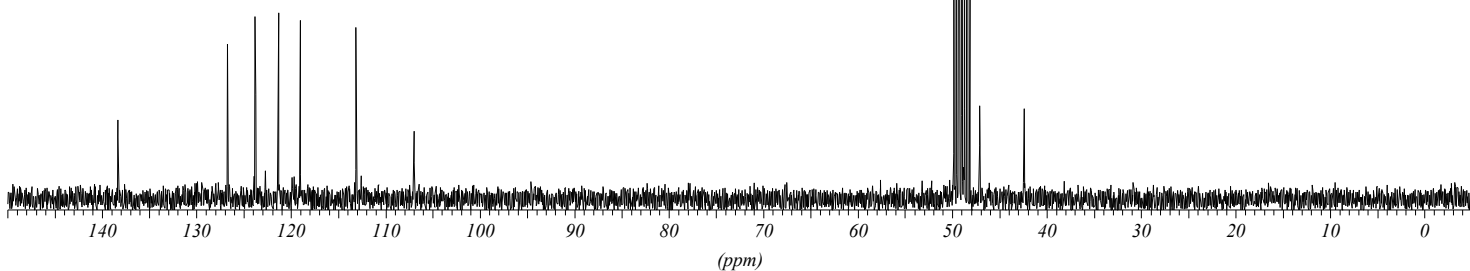



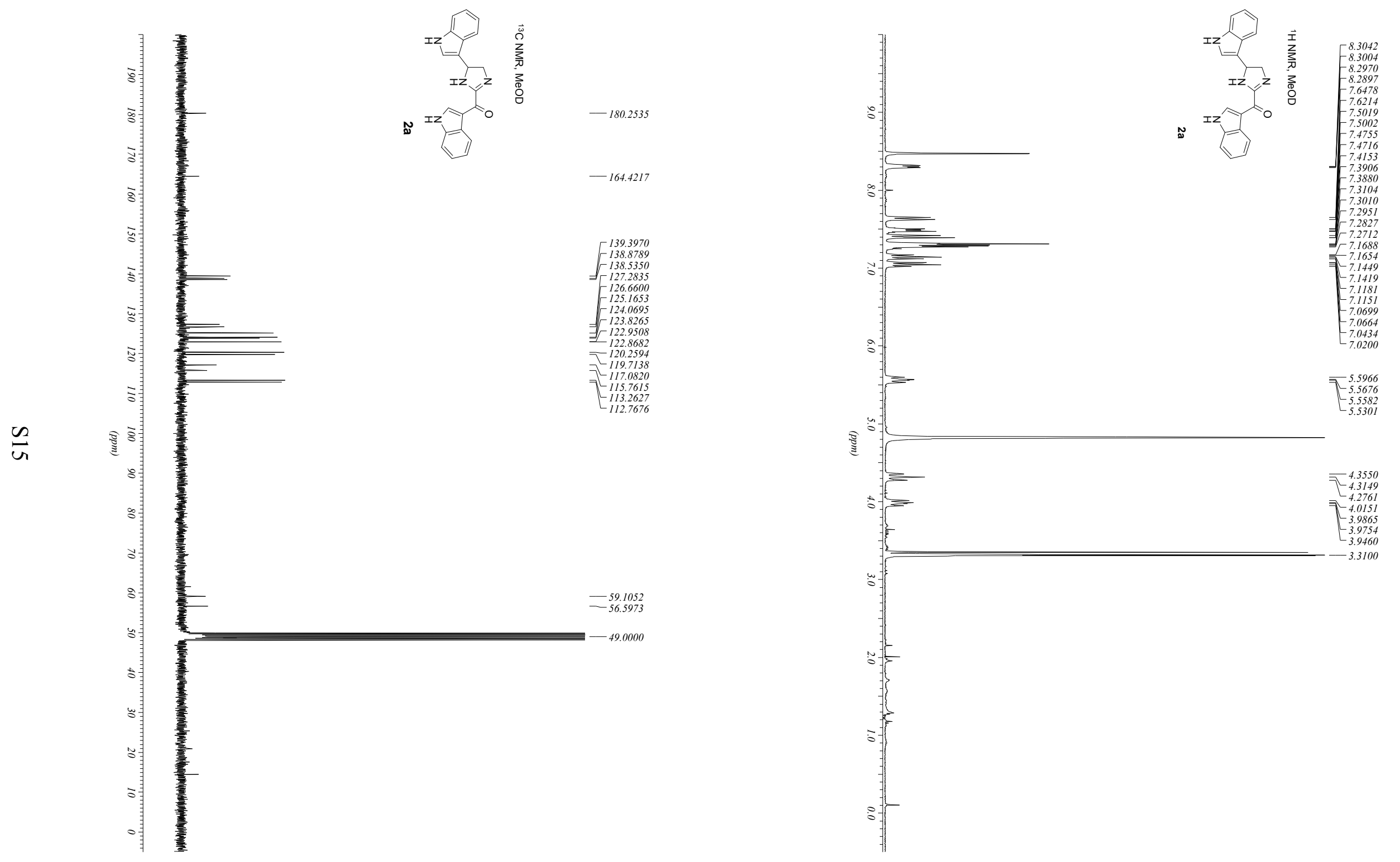


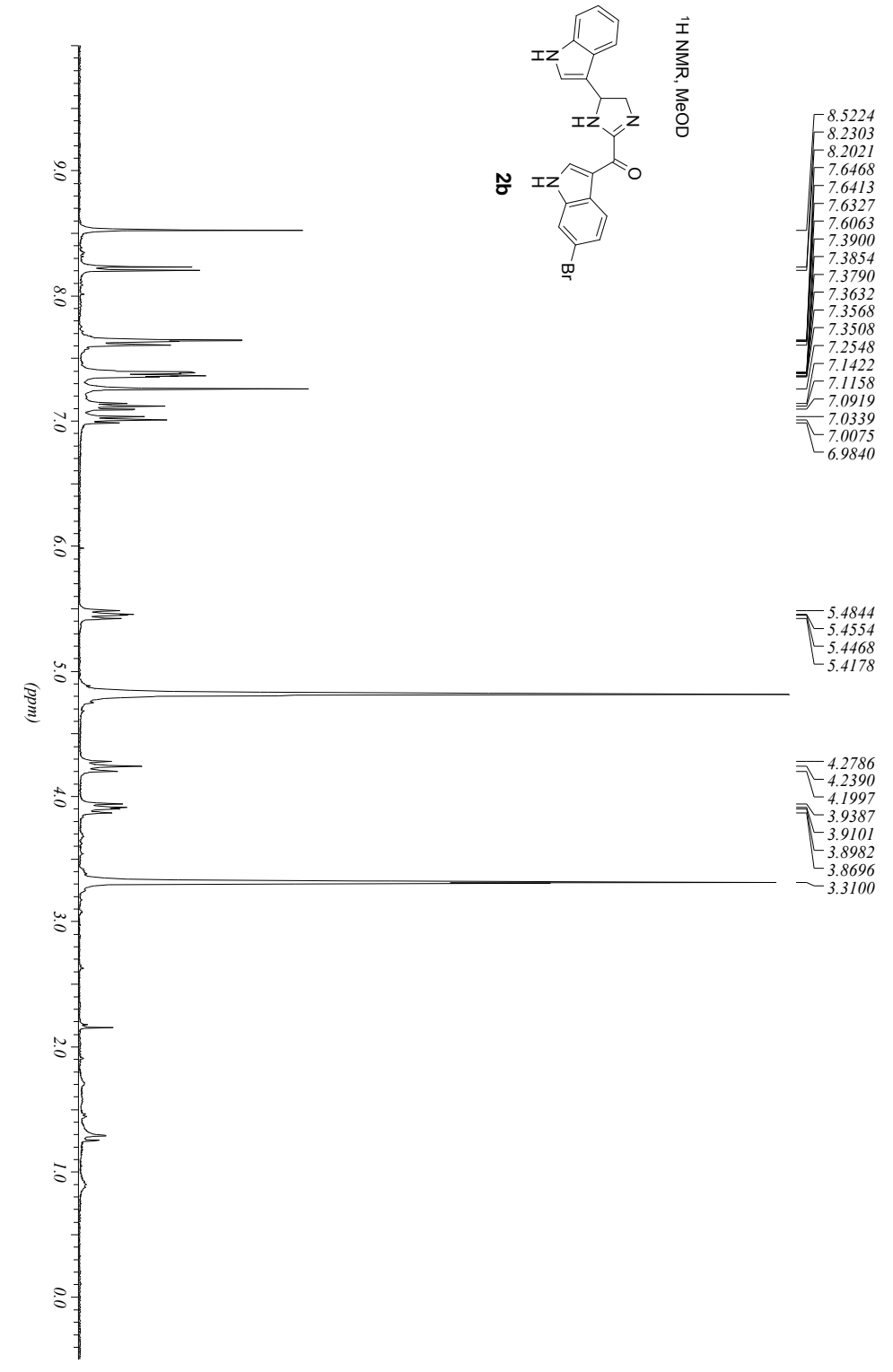



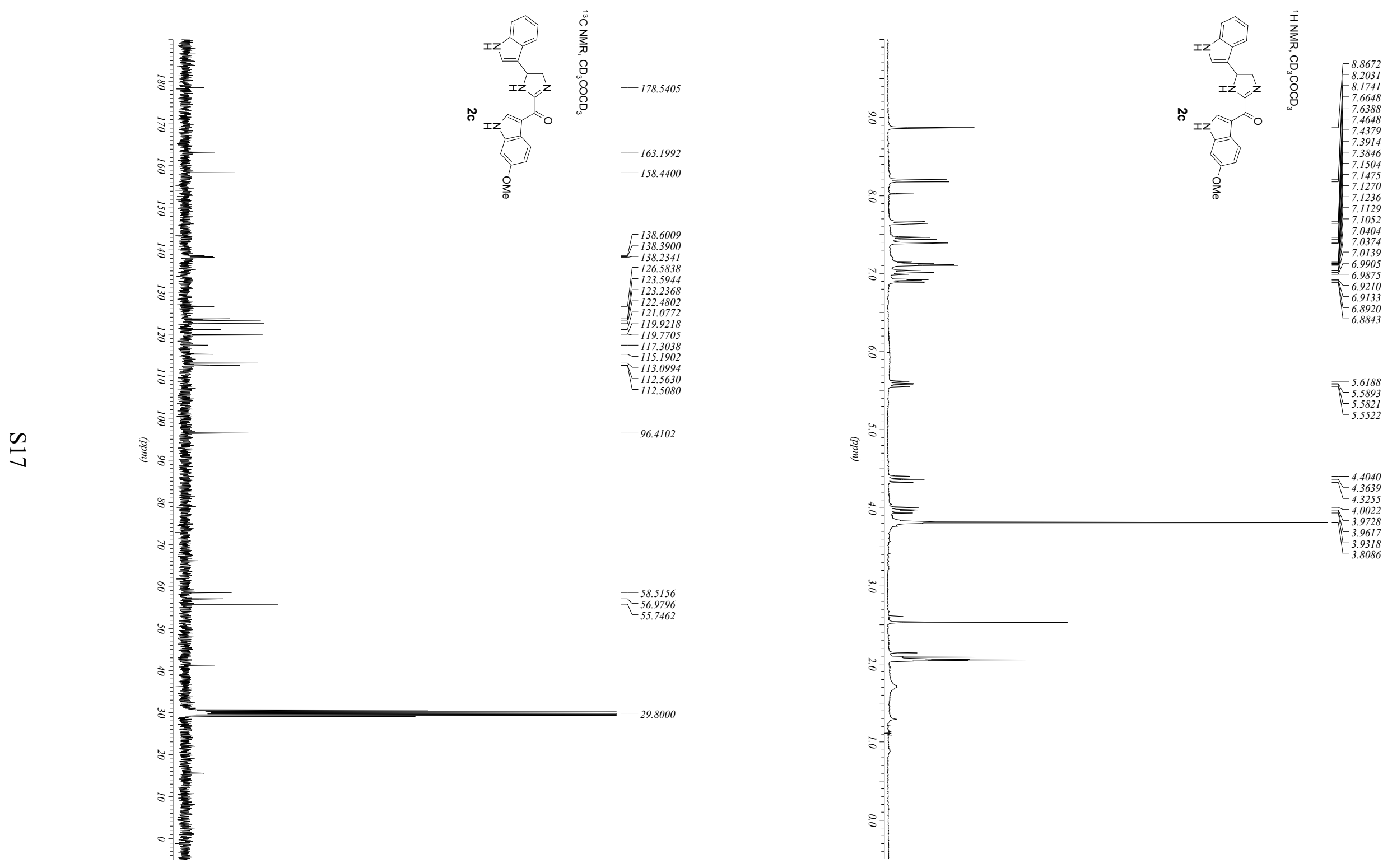


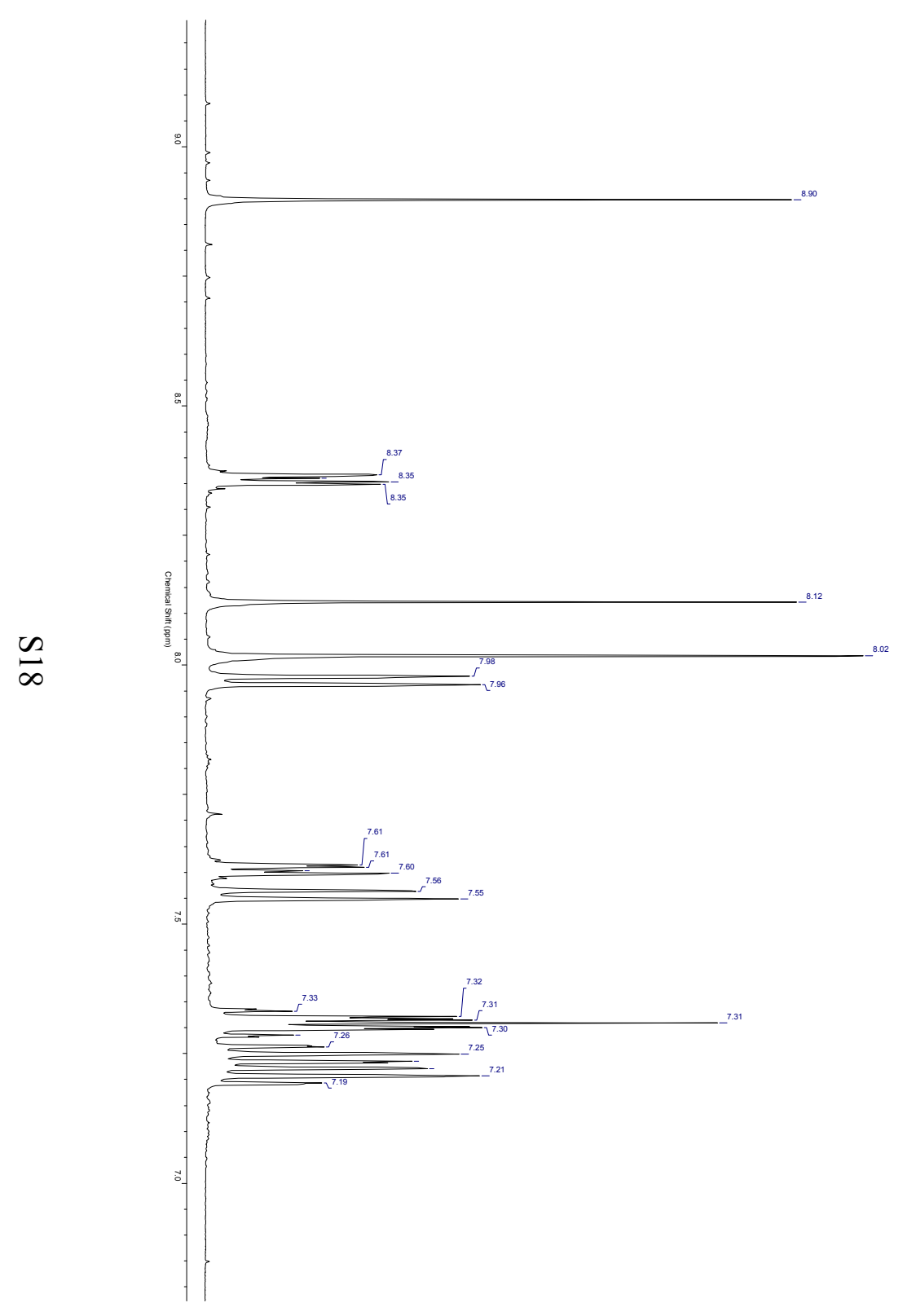

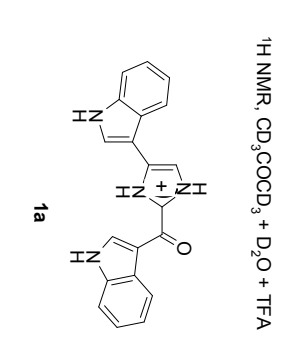

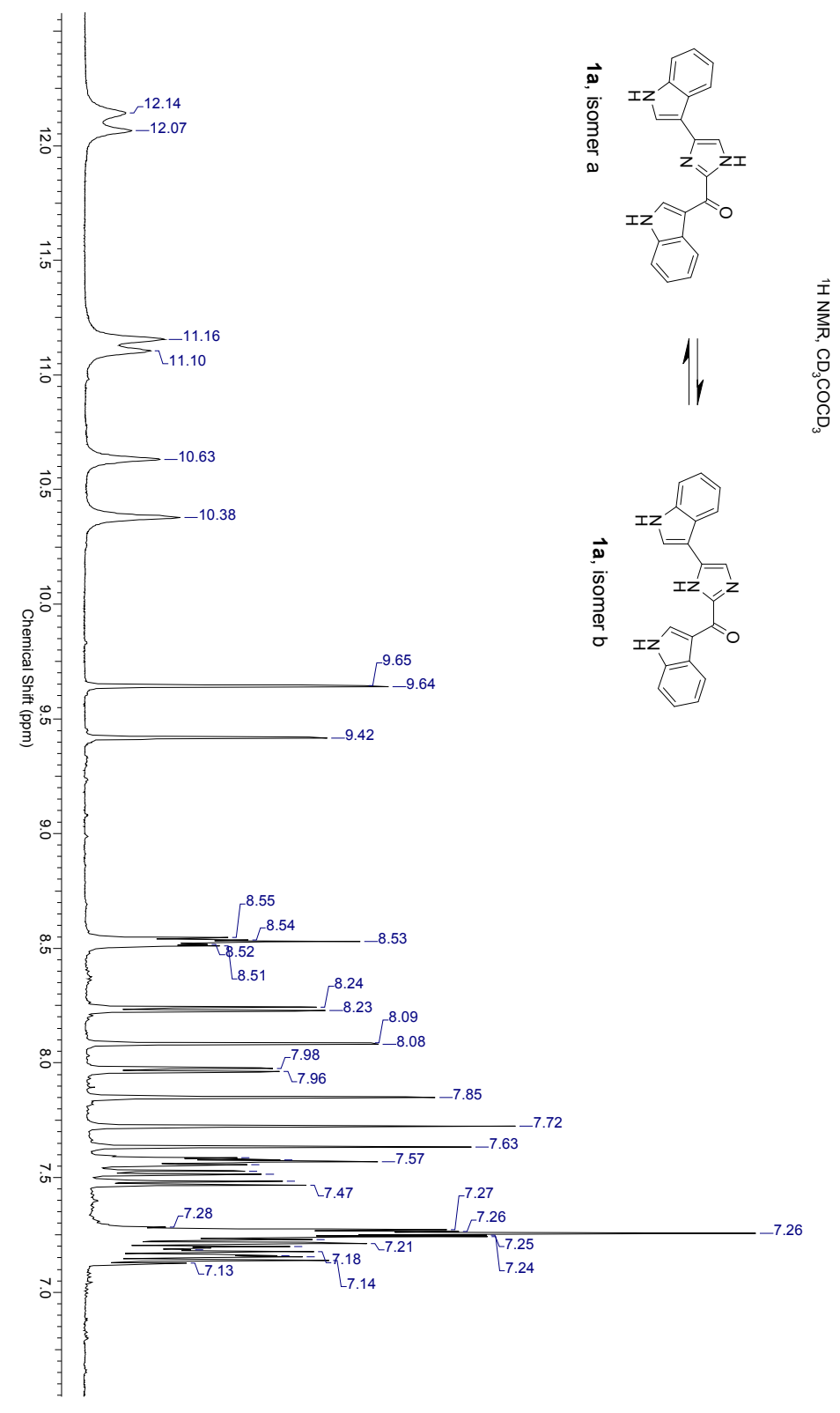



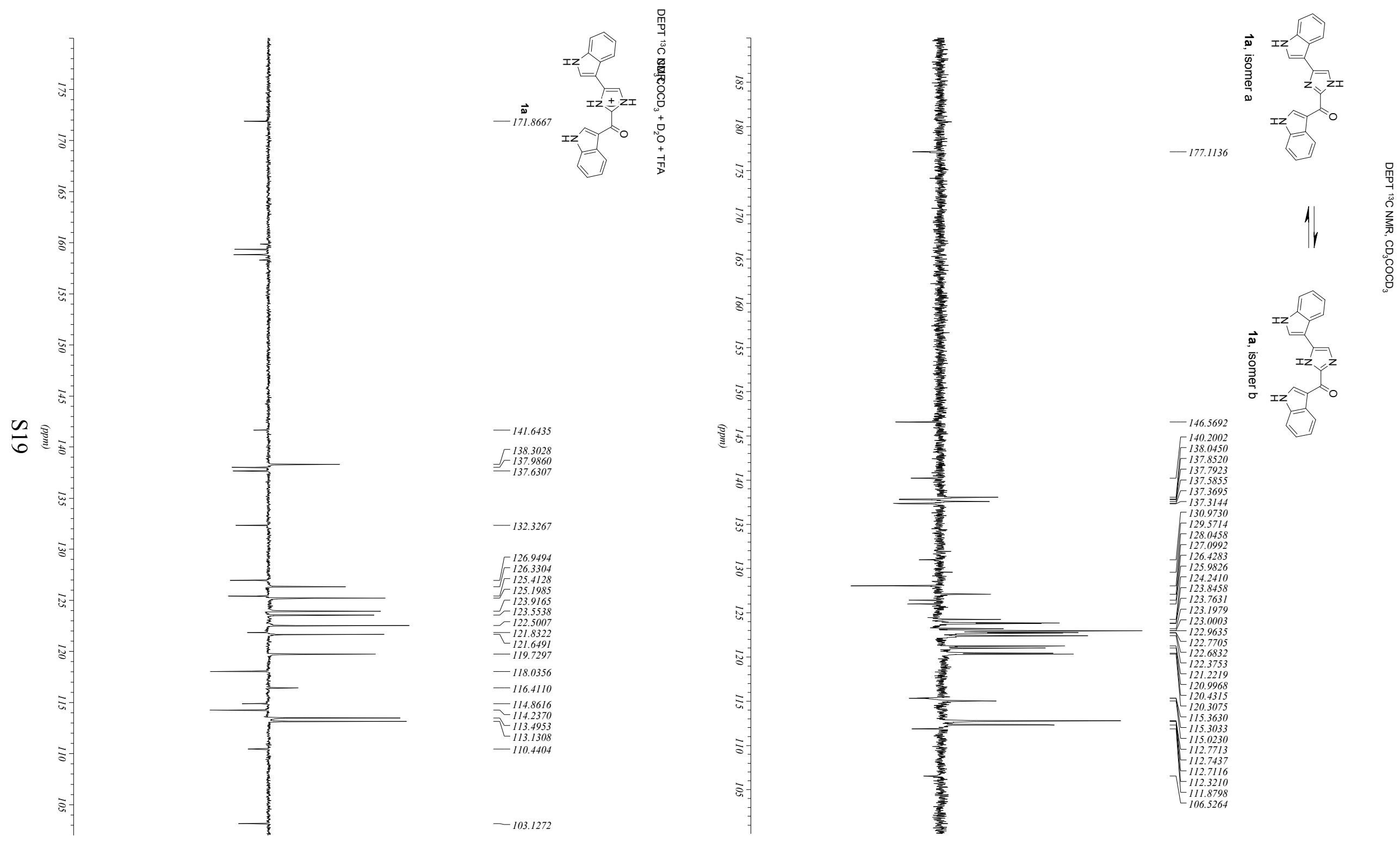


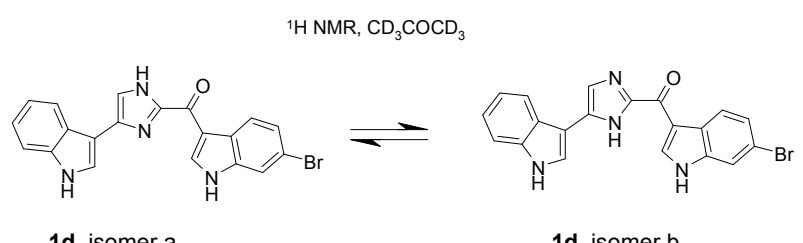

1d, isomer a

1d, isomer b

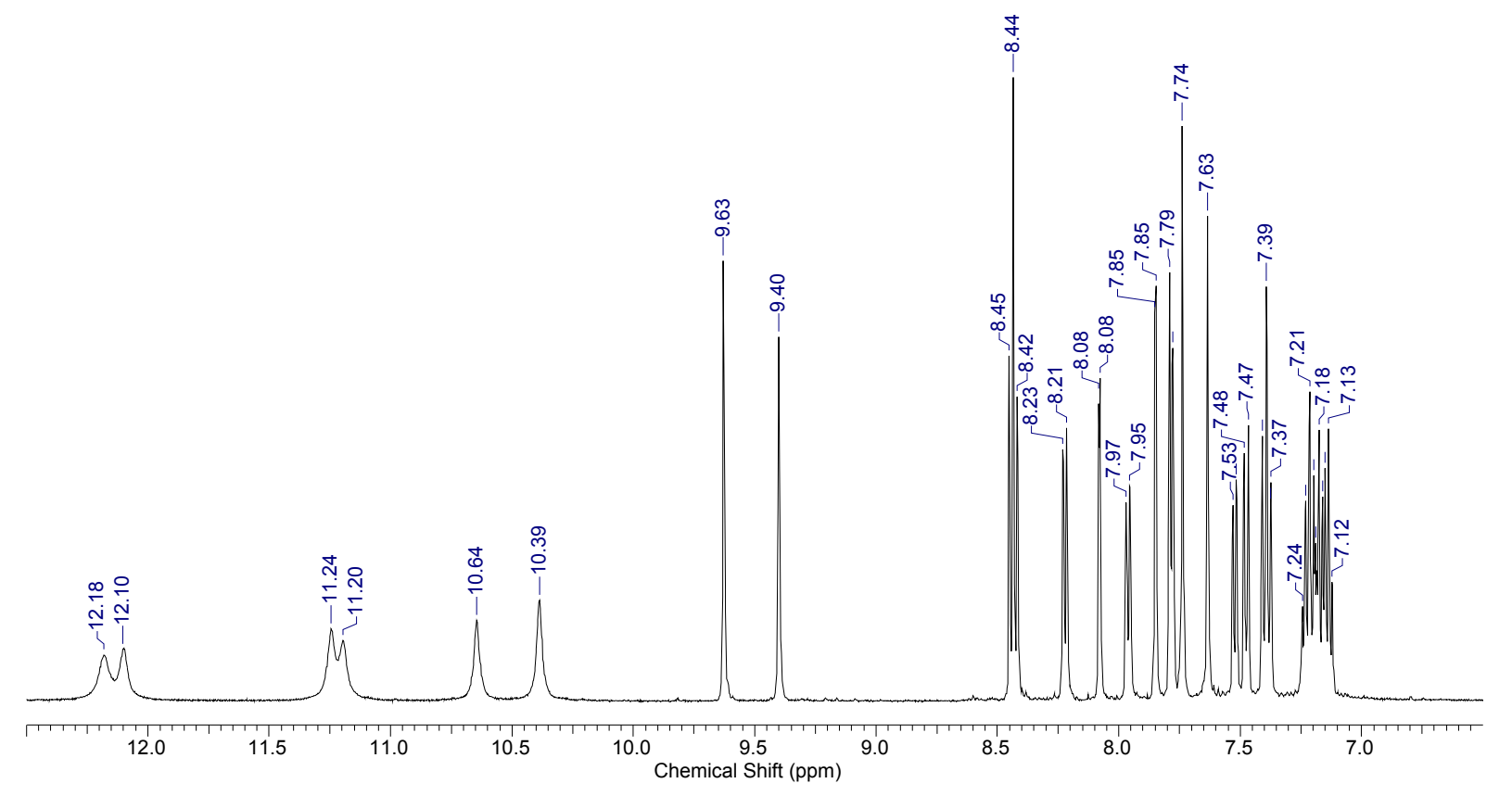

${ }^{1} \mathrm{H} \mathrm{NMR}, \mathrm{CD}_{3} \mathrm{COCD}_{3}+\mathrm{D}_{2} \mathrm{O}+\mathrm{TFA}$

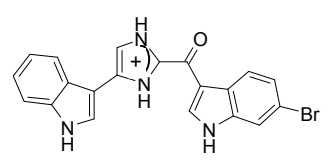

1d

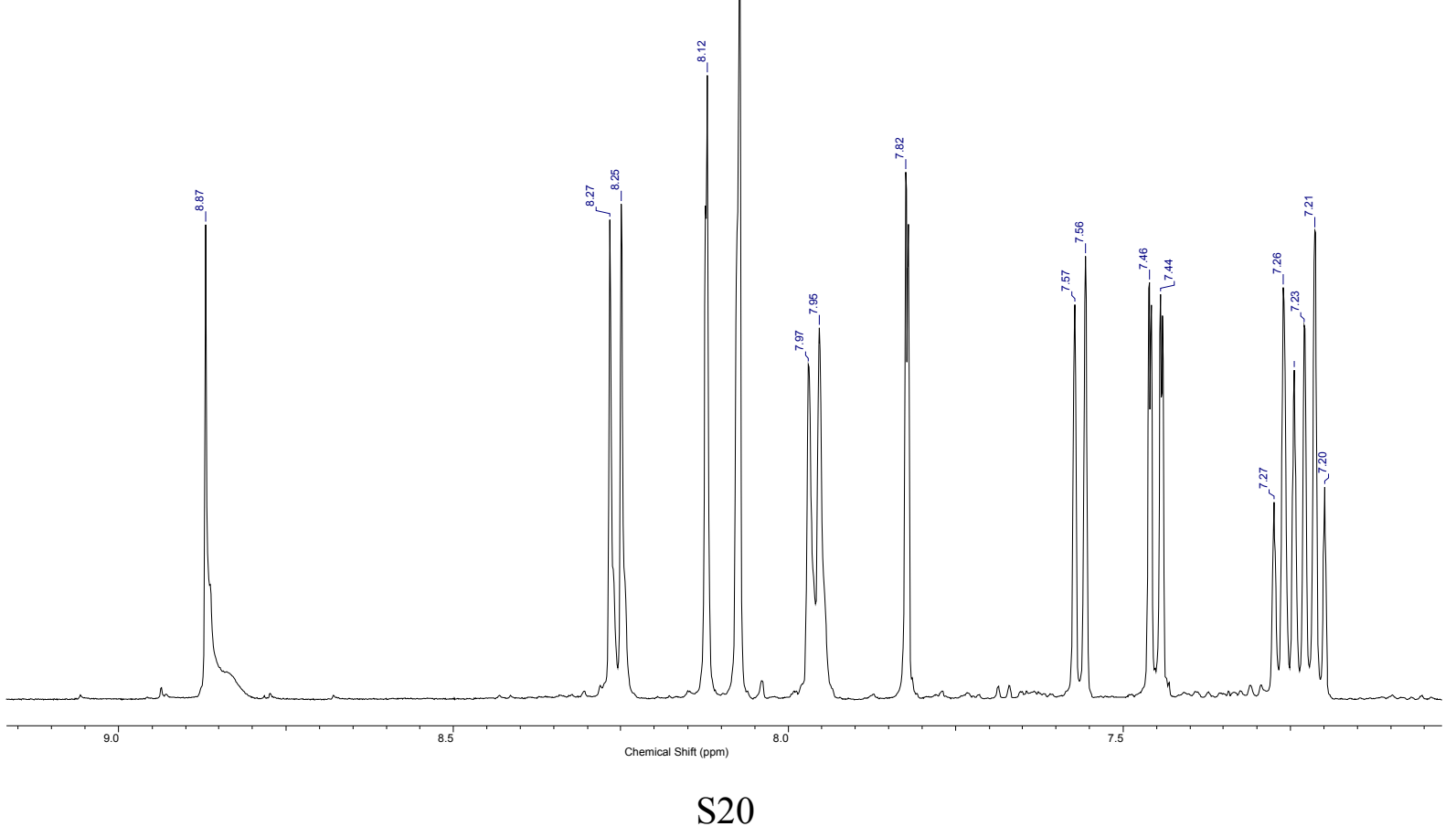



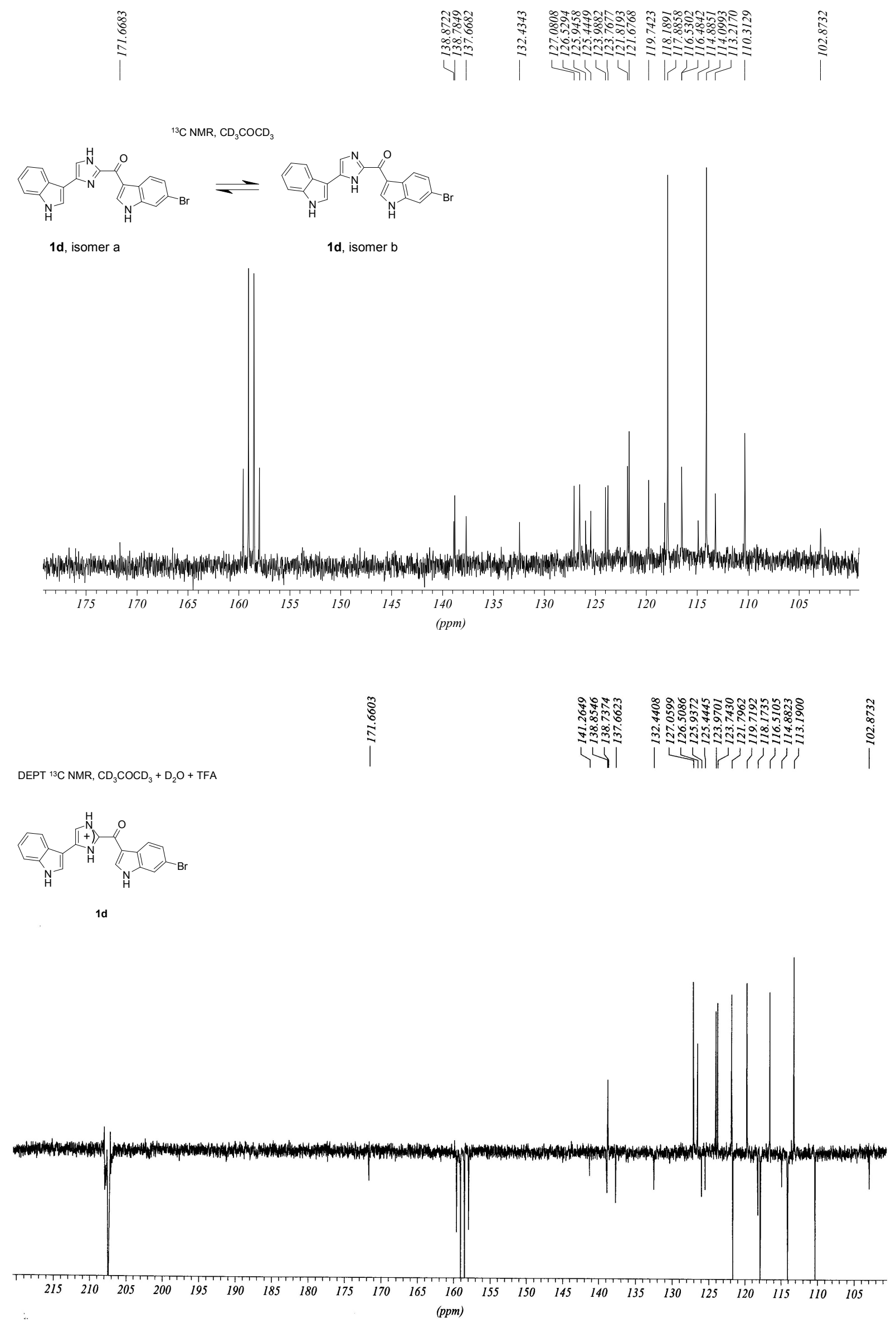

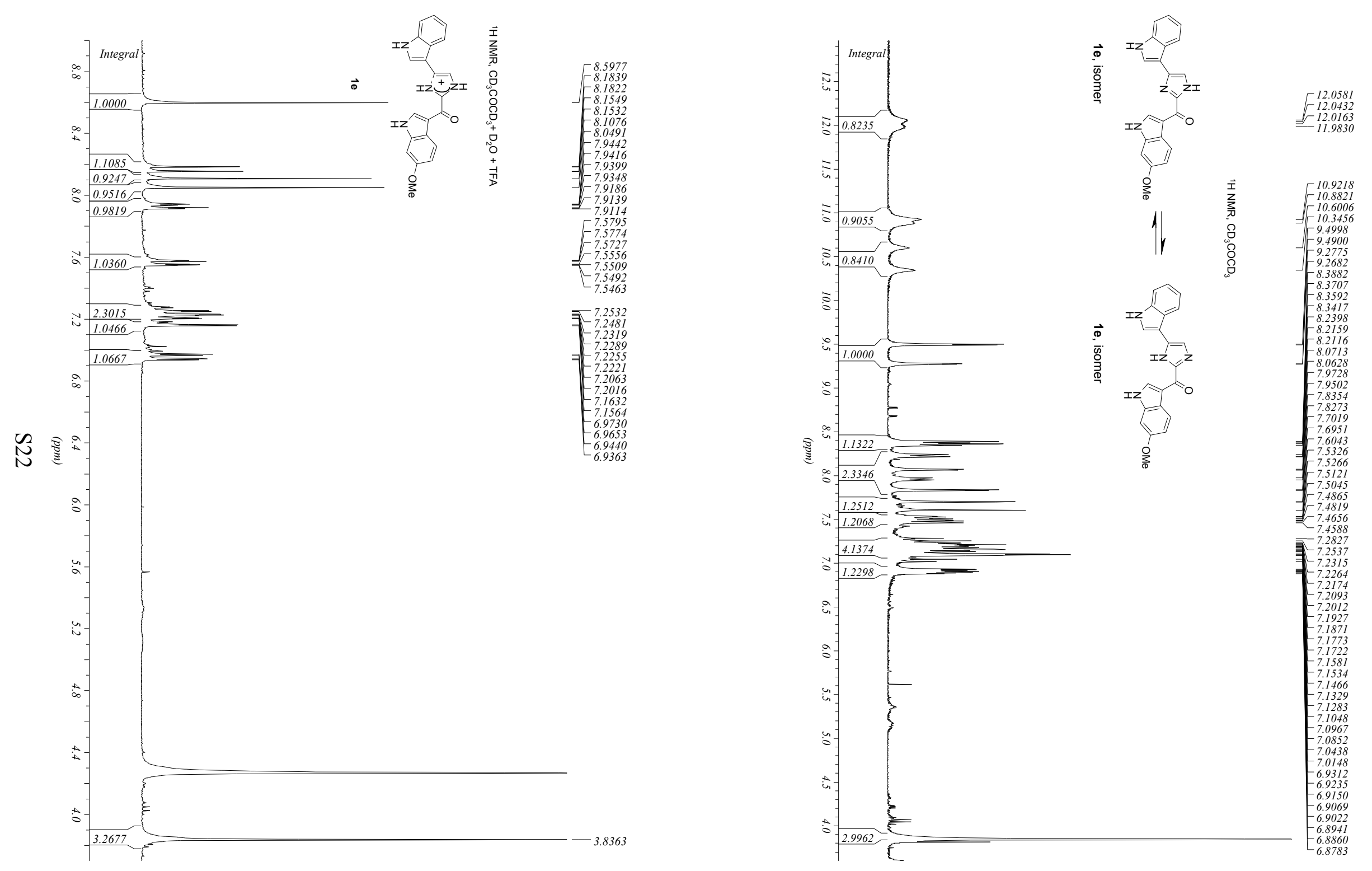


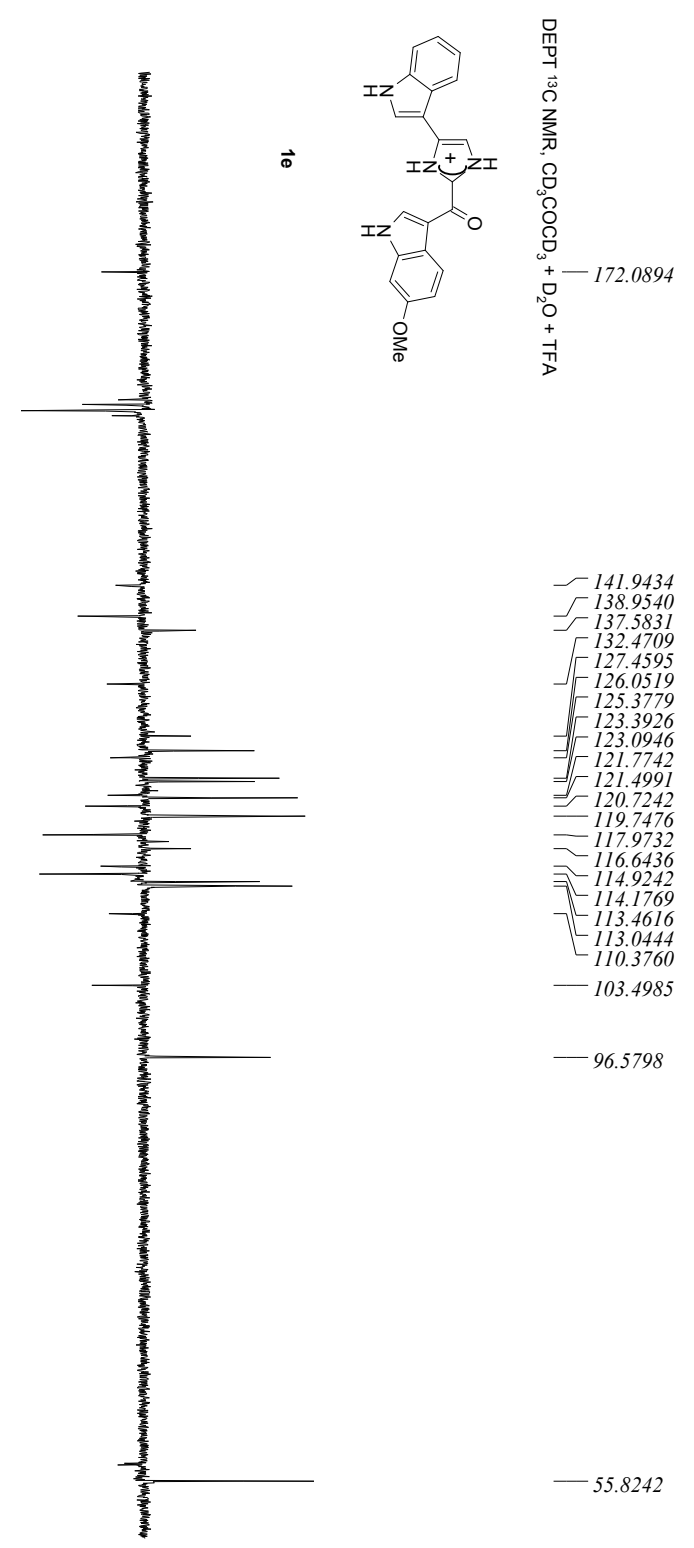

